\title{
Histone Deactylase Inhibitors as Novel Target for Cancer, Diabetes, and Inflammation
}

\author{
Parul Singh and Thirumurthy Madhavan ${ }^{\dagger}$
}

\begin{abstract}
Histone deacetylase (HDACs) is an enzyme family that deacetylates histones and non-histones protein. Availability of crystal structure of HDAC8 has been a boosting factor to generate target based inhibitors. Hydroxamic class is the most studied one to generate potent inhibitors. HDAC class I and class II enzymes are emerging as a therapeutic target for cancer, diabetes, inflammation and other diseases. DNA methylation and histone modification are epigenetic mechanism, is important for the regulation of cellular functions. HDACs enzymes play essential role in gene transcription to regulate cell proliferation, migration and death. The aim of this article is to provide a comprehensive overview about structure and function of HDACs enzymes, histone deacetylase inhibitors (HDACi) and HDACs enzymes as a therapeutic target for cancer, inflammation and diabetes.
\end{abstract}

Key words: HDACs, Enzymes, Inhibitors, Cancer

\section{Introduction}

Histone deacetylase (HDACs) enzymes are present in bacteria, fungi, plants and animals. HDAC is a zinc dependent enzyme. HDAC and HAT (histone acetyl transferase) regulates acetylation, which leads into the post translational modification. Unlike HDAC, HAT enzymes are only present in eukaryotes ${ }^{[1]}$. These two enzymes HDAC and HAT are functionally opposite. Regulation of histone acetylation and deacetylation is an essential part of gene regulation. Acetylation and deacetylation both happens on lysine residues of the Nterminal tail which is present on the surface of nucleosome ${ }^{[2]}$. Histones are the building blocks of chromatin and it's translational modification is responsible for the transcriptional regulation in eukaryotes ${ }^{[1]}$. Positive charge on histone is removed by acetylation. This decreases interaction between $\mathrm{N}$-termini of histones and phosphate groups of DNA which is negatively charged. As a result condensed chromatin is transformed into a relaxed structure, which helps in gene transcription ${ }^{[3]}$,

Department of Bioinformatics, SRM University, SRM Nagar, Kattankulathur Chennai, India

†Corresponding author : thirumurthy.m@ktr.srmuniv.ac.in thiru.murthyunom@gmail.com (Received : January 31, 2013, Revised : March 20, 2013, Accepted : March 25, 2013) while deacetylation increases positive charge on the Ntermini of histones by mediating the removal of epsilonacetyl which is a group of lysine residues. It includes core nucleosomal histones also like H2A, H2B, H3 and H4. In other words, it reverses the outcome of acetylation $^{[1,2]}$. As a result of deacetylation condensed structure of chromatin is found, which prevents accessing of transcriptional factors. Histone deacetylation can also term as hypoacetylation and is associated with repression of gene transcription. There should be a balance between catalytic function of these two enzymes, for normal regulation of gene transcription ${ }^{[3]}$.

HDAC enzymes modulate function of histones and many other proteins; those are involved in regulation of cell survival and proliferation, inflammation, angiogenesis and immunity ${ }^{[4]}$. Histone deacetyltransferases and histone deacetylases are epigenetic regulators. Epigenetic is a field which is concerned with the ability of accessing genes and how it affects biological processes $^{[9]}$. DNA methylation and post translational modification of amino acids in histone proteins considered as a two major biochemical pathways for regulation of epigenetic $^{[20]}$. Gene transcription, mitosis and other cellular process are affected by post translational modification of histone protein ${ }^{[1]}$. Though histone proteins are involved in many post translational modifications such as phosphorylation, acetylation, ubiquitinylation and 

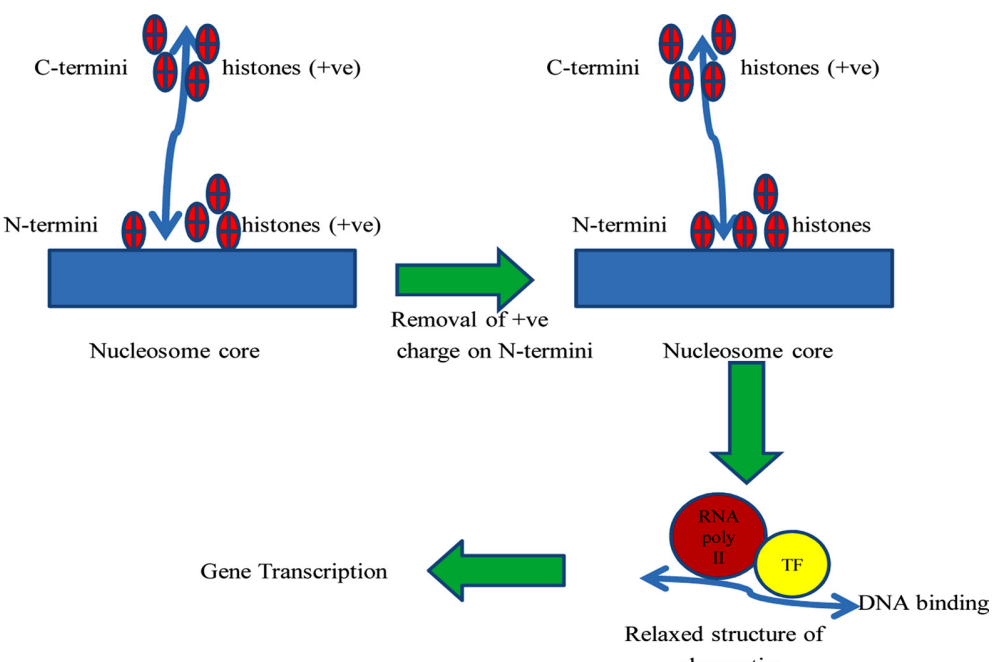

Fig. 1. Flowchart of histone acetylation

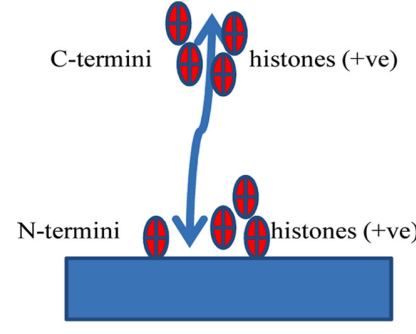

Nucleosome core

Repression of gene transcription

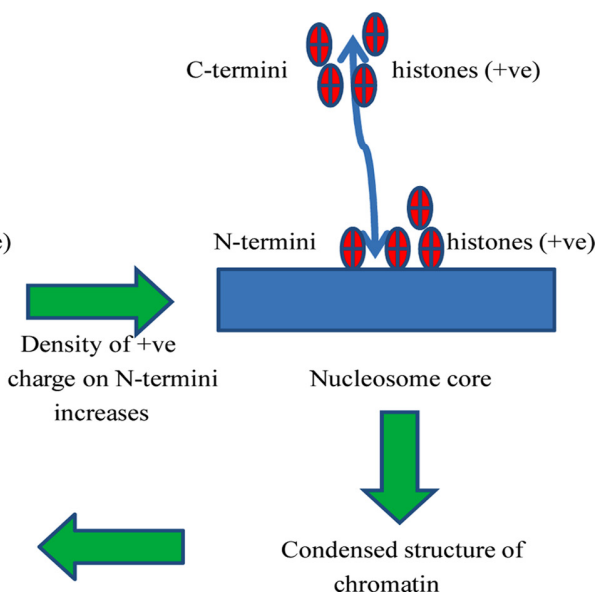

Fig. 2. Flowchart of histone deacetylation

Table 1. Classes of HDAC enzyme

\begin{tabular}{cl}
\hline Class & \multicolumn{1}{c}{ Enzymes } \\
\hline I & HDAC1-3, HDAC8 \\
II(a) & HDAC4, HDAC5, HDAC7, HDAC9 \\
II(b) & HDAC6, HDAC10 \\
III & Sirtuin proteins (1-7) \\
IV & HDAC11 \\
\hline
\end{tabular}

methylation but currently focus is given only on lysine acetylation/deacetylation and methylation/demethylation $^{[4]}$. HDAC enzymes are classified into four classes based on the structure of their accessory domain. There are 18 known human histone deacetylases present. HDAC class I, II and IV are zinc dependent while class III is depend on NAD+ for it's activity ${ }^{[5]}$.

Class I enzymes are distributed in normal tissues. They are highly expressed in lymphoid cell lines and primary tumors ${ }^{[20]}$. Class II enzymes are differentially expressed. HDAC5, HDAC8 and HDAC10 are highly expressed compare to HDAC6 that is why HDAC6 is not considered as an important therapeutic target ${ }^{[6]}$. Class I enzymes have homology to yeast RPD3. Class II (a) enzymes has homology to yeast HDAC1, HDAC6 and HDAC10. Class II enzymes have two catalytic site. Class IV i.e. HDAC11 has conserved residues in its cat- 
alytic center which is shared by both class I and class II enzymes ${ }^{[7]}$.

\section{HDAC Inhibitor}

Finding HDAC inhibitors for HDAC8 is somehow simple in comparison to other HDAC enzymes because active site of HDAC8 is not highly conserved ${ }^{[6]}$. It has been seen that class hydroxamic acid inhibitors, are most studied to find potent HDAC inhibitor ${ }^{[5]}$. A tube like pocket $12 \AA$ long, is present in the catalytic domain of HDACs whose length is equivalent to four to six carbon straight chains ${ }^{[8]}$. An ion $\mathrm{Zn} 2 \mathrm{p}$ is located near the bottom of this enzyme pocket. Most of HDACs enzymes are zinc dependent. This ion is penta-coordinated by two aspartic acids and one histidine ${ }^{[9]}$. The rest of the substrate channel, highly conserved is made up of lipophilic amino acids. Lead compound TSA structure is represented as hydroxamic acid class of HDACs inhibitors which can be divided into three molecular fragment ${ }^{[20]}$. Each fragment interacts with a discrete region of the enzyme pocket. These fragments have zinc binding group (ZBG), cap group and a linker who is connecting to the both $\mathrm{ZBG}$ as well as cap at a proper distance $^{[21]}$. This three fragment concept has been very popular in developing structural analogues of TSA as potent HDACs inhibitors. There are three common structural characteristics are present in all HDAC inhibitors ${ }^{[8]}$.

Availability of crystal structure of HDAC8 has been very useful to develop target based inhibitors. Developing HDAC isoforms to treat certain diseases can provide potent inhibitor with fewer side effects ${ }^{[7]}$. Modification of the capping group, linker and zinc binding group ( $\mathrm{ZBG}$ ) should be individually selective to develop a better HDAC isoforms ${ }^{[8]}$. A better understanding of the binding mode of HDAC inhibitors can be developed by determination of X-ray crystal structure of human HDAC8 with different inhibitors (PDB ID: 1T64, 1T67, 1T69, 1VKG, 1W22, 2VKG, 3F0R, and 3 F07 ${ }^{[20]}$. Focus on HDAC inhibitors has been increased; so many classes of HDAC inhibitors are present now.

In vitro study shows that inhibitors bind directly to the HDAC active site followed by blocking substrate access which causes the accumulation of acetylated histones while In vivo xenograft studies have demonstrated that many of these agents can be effective in the inhibition of tumor growth ${ }^{[21]}$. Crystal structure of HDAC8 (1T64) is present in protein data bank (PDB) but it does not share good sequence or structural similarity with other available HDAC isoforms ${ }^{[24]}$. Earlier molecular dynamics and free energy studies have been given better result for a few selective HDAC inhibitors because active residues are highly conserved in class I HDAC proteins $^{[25]}$. Many HDAC inhibitors with highly varied

Table 2. Common properties of HDAC inhibitors

\begin{tabular}{cll}
\hline S.No. & \multicolumn{1}{c}{ Property } & \multicolumn{1}{c}{ Function } \\
\hline 1 & Zinc binding group & To coordinate with catalytic metal ion \\
2 & Hydrophobic spacer & Bind with residues at the tunnel \\
3 & Hydrophobic cap group & Bind with residues at the active site entrance \\
\hline
\end{tabular}

Table 3. Classes of HDAC inhibitors

\begin{tabular}{lll}
\hline \multicolumn{1}{c}{ Class } & \multicolumn{1}{c}{ Inhibitors name } & \multicolumn{1}{c}{ Stage } \\
\hline Hydroxamic acid & Vorinostat, Belinostat & approved \\
& Panobinostat (LBH589), & Phase II development \\
& ITF-2357, PXD101 & \\
Benzamide & MS-275, Mocetinostat (MGCD0103) & Active development \\
Cyclic peptide & Depsipeptide & Active development \\
Short chain fatty acid & Valproic acid, Butyrate & Active development \\
Bicyclic depsipeptide & Romidepsin & Clinical development \\
- & Entinostat (SNDX-275) & Clinical development \\
- & B lactams & Clinical development \\
\hline
\end{tabular}


activities and chemotypes can be easily identified by Inhouse build Medichem database which is a very powerful resource. HDAC Class I and II enzymes are emerging as a therapeutic target for cancer, diabetes, inflammation and other diseases ${ }^{[26]}$.

\section{Cancer/anti-tumor Activity}

Cancer is characterized by out of control cell growth. Damaged cells divide uncontrollably to form masses of tissue called tumors (except in the case of leukemia where normal blood function is prohibited by abnormal cell division in the blood stream ${ }^{[23]}$. There are more than 100 different types of cancer present and each one is classified by the type of cell that is initially affected $^{[22]}$. Histone deactylase inhibitors (HDACIs) are showing good activity in inducing cell-cycle arrest, apoptosis, differentiation, inhibiting invasion, migration, and angiogenesis in many cancer cell lines ${ }^{[24]}$. Theses inhibitors have shown antitumor activity in animal model by inhibiting growth of tumor ${ }^{[25]}$.

\section{Vorinostat}

The FDA has approved vorinostat in $2006^{[22]}$. Vorinostat has potent anti proliferative activity in Hodgkin lymphoma derived cell ${ }^{[26]}$. It inhibits enzymes of both classes HDAC I and HDAC II. Vorinostat has good synergy with chemotherapy ${ }^{[3]}$. Combined result of phase I and phase II has proved that Vorinostat induced cell cycle arrest and apoptosis ${ }^{[4]}$.

\section{Belinostat}

This inhibitor is in clinical development. Belinostat can be taken via many routes like intravenous administration, oral administration and continuous intravenous ${ }^{[27]}$. It belongs to hydroxamic acid derived class of HDAC inhibitor, which is considered to be most studied class for HDAC inhibitors. Belinostat has demonstrated good results in some solid tumors ${ }^{[29]}$.

\section{Romidepsin}

Romidepsin inhibits enzymes of both classes HDAC I and HDACII. It is isolated from the bacterium Chromobacterium violaceum. The FDA has approved
Romidepsin for the treatment of cutaneous T-cell lymphoma $^{[31]}$. Romidepsin inhibits hypoxia induced angiogenesis along with cell differentiation, cell cycle arrest and apoptosis ${ }^{[32]}$. The clinical trial of Romidepsin was done on 167 patients $^{[33]}$. Duringg clinical trial some adverse effects also appeared like infection, vomiting, nausea, fatigue and anorexia, decreased appetite, decreased red blood cell count and decreased white blood cell components ${ }^{[34]}$.

\section{Panobinostat}

Panobinostat has shown anti tumor activity with Hodgkin lymphoma, acute myeloid leukemia and multiple myeloma ${ }^{[35]}$. Panobinostat has shown good activity with HDAC classes I, II and IV. Panobinostat acetylation has induced $\mathrm{H}$ and $\mathrm{H} 4$ histones ${ }^{[36]}$. As a result $\mathrm{p} 21$ level increases which disrupts chaperon function, followed by apoptosis acute leukemia cells ${ }^{[37]}$. Panobinostat has shown good results in phase I and phase II for the median age of participants and many of them were from stage III/IV ${ }^{[38]}$.

\section{Diabetes Mellitus}

Diabetes mellitus can also be termed as diabetes, this is a shortened version. In the $17^{\text {th }}$ century it was known as the "pissing evil" because of the presence of excess sugar in blood as well as in the urine ${ }^{[11]}$. Diabetes is one of the fastest growing long term diseases. If this trend will continue then one in three Americans would be diabetic by 2050 . There are mainly two type of diabetes are present, type 1 and type $2^{[10]}$. Type 1 diabetes can also be termed as insulin dependent diabetes while type 2 diabetes can be termed as non-insulin dependent diabetes. Around $75 \%$ of people with diabetes have type 2 diabetes $^{[11]}$. HDAC inhibition is having a specific impact on overall immune system in relation to T1D and T2D. HDACi regulates both innate as well as adaptive immune responses ${ }^{[12]}$. Enzyme cyclooxygenase (COX)-2 in monocytes isolated from patients with T1D or T2D. Histone $\mathrm{H} 3$ is hyperacetylated in the promoters of tumor necrosis factor- $\alpha(\mathrm{TNF} \alpha)^{[13]}$. Both are having importance of the activity of HATs and HDACs in the expression of proinflammatory genes in monocytes $^{[12,13]}$. It has been found that hyperacetylation is a resultenet of hyperglycemia but this is not a reason of 
diabetes $^{[28]}$. Histone acetylation is triggered by high concentration of glucose and HDAC inhibitor trichostatin A from diabetics ${ }^{[11]}$. Production of inflammatory cytokines IL-1 $\beta$ and TNF $\alpha$ was triggered by high glucose concentrations through activation of $\mathrm{NFêB}^{[16]}$. Activity of NFêB can be enhanced by HAT and TSA over expression and can be reversed by over expression of HDAC1- $6^{[14]}$. Side effects of HDACi is based on it's concentration. High concentration will lead to more side effects while low concentration will have minimum side effects but low concentration is related to anti inflammatory responses ${ }^{[15]}$. On conclusion, the effects of HDAC inhibition with respect to diabetes are not clarified and further investigation is needed to reveal the dose-response relationships on cytokine production from monocytes.

\section{Inflammation}

A response of body tissues to injury or irritation; characterized by pain and swelling and redness and heat is inflammation ${ }^{[17]}$. It is not similar to infection even if it is caused by infection. Histone deacetylase inhibitors (HDACi) have anti-inflammatory properties according to the Molecular medicine ${ }^{[18]}$. HDACi have shown good results in plenty of animal and cellular models of inflammatory diseases like arthritis, septic shock, graft versus host disease and inflammatory bowel disease ${ }^{[19]}$. According to the recent advances in phylogenetic analysis, HDACs regulate the activity of wide range of non histone proteins ${ }^{[30]}$. Currently 3,600 acetylation sites on 1,750 proteins have been found and among them only 61 were on histones. Inflammation nuclear factor (NF)$\kappa \mathrm{B}$ is a main transcription factor which is regulated by acetylation ${ }^{[39]}$. Finding selective mode of action for HDACi in reducing inflammation-related pathologies is a tough job. Inhibitors pan-HDAC inhibits Th1 and Th17 development to suppress the destructive capacity of the innate immune system but non-immune cells also contribute $^{[40]}$. So, more potent HDAC inhibitors are required. Understanding for HDAC functions inmmunerelated pathways should be widely emerges. Class I HDACs are involved in negatively regulating inflammatory cytokine production and in promoting antiviral responses. Class II(a) HDACs control lymphocyte development and function. In present scenario, there can be chances for the development of specific inhibi- tors of class I HDACs, HDAC6, HDAC7, HDAC9 and HDAC11 for immune-related applications.

\section{Conclusion}

HDACs appear to have great impact on epigenetic mechanism and further play key role in the regulation of gene expression. Although few studies indicated that HDACi has reported minor side effects. This review provides evidence that supports the role of HDACs in diabetes, cancer and inflammation. It also describes strong rational to continue preclinical studies and initiate clinical trials of HDAC inhibitors. Currently many efforts are being made to develop potent and stable HDACi. In the future, target based HDACi might rise who's therapeutic range will be wide.

\section{References}

[1] A. J. M. De Ruijter, A. H. Van Gennip, H. N. Caron, S. Kemp, and A. B. P. Van Kuilenburg, "Histone deacetylases (HDACs): characterization of the classical HDAC family", Biochem. J., Vol. 370, pp. 737-749, 2003.

[2] K. Ito, P. J. Barnes, and I. M. Adcock, "Glucocorticoid receptor recruitment of histone deacetylase 2 inhibits interleukin-1beta-induced histone H4 acetylation on lysines 8 and 12", Mol. Cell. Biol., Vol. 20, pp. 6891-6903, 2000.

[3] D. J. Witter, S. Belvedere, L. Chen, J. Paul Secrist, R. T. Mosleyd, and T. A. Millera, "Benzo[b]thiophenebased histone deacetylase inhibitors", Bioorg. Med. Chem. Lett., Vol. 17, pp 4562-4567, 2007.

[4] T. Sundarapandian, J. Shalini, S. Sugunadevi, and L. K. Woo, "Docking-enabled pharmacophore model for histone deacetylase 8 inhibitors and its application in anti-cancer drug discovery", J. Mol. Graph. Model., Vol. 29, pp. 382-395, 2010.

[5] S. Thangapandian, S. John, S. Sakkiah, and K. W. Lee, "Ligand and structure based pharmacophore modeling to facilitate novel histone deacetylase 8 inhibitor design", Eur. J. Med. Chem., Vol. 45, pp. 4409-4417, 2010.

[6] D. J. Witter, S. Belvedere, L. Chen, J. P. Secrist, R. T. Mosleyd, and T. A. Miller, "Benzo[b]thiophenebased histone deacetylase inhibitors", Bioorg. Med. Chem. Lett., Vol. 17, pp. 4562-4567, 2007.

[7] S. Vadivelan, B.N. Sinha, G. Rambabu, K. Boppana, and S.A.R.P. Jagarlapudi, "Pharmacophore model- 
ing and virtual screening studies to design some potential histone deacetylase inhibitors as new leads", J. Mol. Graph. Model., Vol. 26, pp. 935-946, 2008.

[8] Y. Chen, H. Li, W. Tang, C. Zhu, Y. Jiang, J. Zou, Q. Yu and Q. You, "3D-QSAR studies of HDACs inhibitors using pharmacophore-based alignment", Eur. J. Med. Chem., Vol.44, pp. 2868-2876, 2009.

[9] R. Heinke, L. Carlino, S. Kannan, M. Jung, and W. Sippl, "Computer- and structure-based lead design for epigenetic targets", Bioorg. Med. Chem., Vol. 19, pp. 3605-3615, 2011.

[10] D. P. Christensen, M. Dahllöf, M. Lundh, D. N. Rasmussen, M. D. Nielsen, N. Billestrup, L. G. Grunnet, and T. M. Poulsen, "Histone deacetylase (HDAC) inhibition as a novel treatment for diabetes mellitus", doi: 10.2119/molmed.2011.00021

[11] S. Balasubramanian, J. Ramos, W. Luo, M. Sirisawad, E. Verner, and J. J. Buggy, "A novel histone deacetylase 8 (HDAC8)-specific inhibitor PCI34051 induces apoptosis in T-cell lymphomas Leukemia 22", doi: 10.1038/leu.2008.9

[12] H. S. Suh, S. Choi, P. Khattar, N. Choi, and S. C. Lee, "Histone deacetylase inhibitors suppress the expression of inflammatory and innate immune response genes in human microglia and astrocytes", J. Neuroimmune Pharmacol., Vol. 5, pp. 521-32, 2010.

[13] F. Miao, I. G. Gonzalo, L. Lanting, and R. Natarajan, "In vivo chromatin remodeling events leading to inflammatory gene transcription under diabetic conditions", J. Biol. Chem., Vol. 279, pp. 1809118097, 2004.

[14] N. Shanmugam, M. A. Reddy, M. Guha, and R. Natarajan, "High glucose-induced expression of proinflammatory cytokine and chemokine genes in monocytic cells", Diabetes, Vol. 52, pp. 1256-64, 2003.

[15] M. A. Halili, M. R. Andrews, L. I. Labzin, K. Schroder, G. Matthias, C. Cao, E. Lovelace, R. C. Reid, G. T. Le, D. A. Hume, K. M. Irvine, P. Matthias, D. P. Fairlie, and M. J. Sweet, "Differential effects of selective HDAC inhibitors on macrophage inflammatory responses to the Toll-like receptor 4 agonist LPS”, J. Leukoc. Biol., Vol. 87, pp. 110314, 2010.

[16] F. Leoni, G. Fossati, E. C. Lewis, J. K. Lee, G. Porro, P. Pagani, D. Modena, M. L. Moras, P. Pozzi, L. L. Reznikov, B. Siegmund, G. Fantuzzi, C. A. Dinarello, and Paolo Mascagni, "The histone deacetylase inhibitor ITF2357 reduces production of proinflammatory cytokines in vitro and systemic inflammation in vivo", Mol. Med., Vol. 11, pp. 1$15,2005$.

[17] M. A. Halili, M. R. Andrews, M. J. Sweet, and D. P. Fairlie, "Histone deacetylase inhibitors in inflammatory disease", Curr. Top. Med. Chem., Vol. 9, pp. 309-319, 2009.

[18] I. V. Gregoretti, Y. M. Lee, and H. V. Goodson, "Molecular evolution of the histone deacetylase family: functional implications of phylogenetic analysis", J. Mol. Biol., Vol. 338, pp. 17-31, 2004.

[19] C. Choudhary, C. Kumar, F. Gnad , M. L. Nielsen, M. Rehman, T. C. Walther, J. V. Olsen, and M. Mann, "Lysine acetylation targets protein complexes and co-regulates major cellular functions", Science, Vol. 325, pp. 834-840, 2009.

[20] A. Vannini, C. Volpari, P. Gallinari, P. Jones, M. Mattu, A. Carfí, R. D. Francesco, C. Steinku“hler, and S. D. Marco, "Substrate binding to histone deacetylases as shown by the crystal structure of the HDAC8-substrate complex", EMBO reports, Vol. 8, pp. 9, 2007.

[21] S. Timmermann, H. Lehrmann, A. Polesskaya, and A. Harel-Bellan, "Histone acetylation and disease", Cell. Mol. Life Sci., Vol. 58, pp. 728-736, 2001.

[22] E. D. Gennaro, F. Bruzzese1, M. Caraglia, A. Abruzzese, and A. Budillon, "Acetylation of proteins as novel target for antitumor therapy", Amino Acids, Vol. 26, pp. 435-441, 2004.

[23] M. Haberland, M. H. Mokalled, R. L. Montgomery, and E. N. Olson, "Epigenetic control of skull morphogenesis by histone deacetylase 8", Gene. Dev., Vol. 23, pp 1625-1630, 2009.

[24] M. Dokmanovic and P. A. Marks, "Prospects: histone deacetylase inhibitors", J. Cell. Biochem., Vol. 96, pp. 293-304, 2005.

[25] B. E. Morrison, N. Majdzadeh, and S. R. D. Mello, "Histone deacetylases: Focus on the nervous system", Cell. Mol. Life Sci., Vol. 64, pp. 2258-2269, 2007.

[26] T. A. McKinsey, "Isoform-selective HDAC inhibitors: Closing in on translational medicine for the heart”, J. Mol. Cell. Cardiol., Vol. 51, pp. 491-496, 2011.

[27] D. M. Fass, S. A. Reis, B. Ghosh, K. M. Hennig, N. F. Joseph d, W. N. Zhao, T. J.F. Nieland, J. S. Guan, C. E. G. Kuhnle, W. Tang, D. D. Barker, R. Mazitschek, S. L. Schreiber, L. H. Tsai, and S. J. H. Crebinostat, "A novel cognitive enhancer that inhibits histone deacetylase activity and modulates chromatin-mediated neuroplasticity", Neuropharmacology, 
Vol. 64, pp. 81-96, 2013.

[28] X. Wanga, X. Weia, Q. Pangb, and F. Yia, "Histone deacetylases and their inhibitors: molecular mechanisms and therapeutic implications in diabetes mellitus", Acta Pharmaceutica Sinica B, Vol. 2, pp. 387-395, 2012.

[29] T. Suzuki, A. Matsuura, A. Kouketsu, H. Nakagawa, and N. Miyata, "Identification of a potent nonhydroxamate histone deacetylase inhibitor by mechanism-based drug design”, Bioorg. Med. Chem. Lett., Vol. 15, pp. 331-335, 2005.

[30] M. R. Shakespear, M. A. Halili, K. M. Irvine, D. P. Fairlie, and Matthew J. Sweet, "Histone deacetylases as regulators of inflammation and immunity", Trends Immunol., Vol. 32, pp. 335-343, 2011.

[31] O. Witt and R. Lindemann "HDAC inhibitors: Magic bullets, dirty drugs or just another targeted therapy", Cancer Lett., Vol. 280, pp. 123-124, 2009.

[32] T. Suzuki, A. Kouketsu, A. Matsuura, A. Kohara, S. I. Ninomiya, K. Kohdaa, and Naoki Miyataa, "Thiol-based SAHA analogues as potent histone deacetylase Inhibitors", Bioorg. Med. Chem. Lett., Vol. 14, pp. 3313-3317, 2004.

[33] S. E. Choi, S. V.W. Weerasinghe, and M. K. H. Pflum, "The structural requirements of histone deacetylase inhibitors: suberoylanilide hydroxamic acid analogs modified at the $\mathrm{C} 3$ position display isoform selectivity", Bioorg. Med. Chem. Lett., Vol. 21, pp. 6139-6142, 2011.

[34] D. Ling, G. M. Marshall, P. Y. Liu, N. Xu, C. A. Nelson, S. E. Iismaa, and T. Liu, "Enhancing the anticancer effect of the histone deacetylase inhibitor by activating transglutaminase", Eur. J. Cancer, Vol. 48, pp. 3278-3287, 2012.

[35] H. Wang, Z. Y. Lim, Y. Zhou, M. Ng, T. Lu, K. Lee, K. Sangthongpitag, K. C. Goh, X. Wangb, X. Wub, H. H. Khng, S. K. Goh, W. C. Ong, Z. Bonday, and E. T. Sun, "A acylurea connected straight chain hydroxamates as novel histone deacetylase inhibitors: synthesis, SAR, and in vivo antitumor activity”, Bioorg. Med. Chem. Lett., Vol. 20, pp. 33143321, 2010.
[36] A. Wahhab, D. Smil, A. Ajamian, M. Allan, Y. Chantigny, E. Therrien, N. Nguyen, S. Manku, S. Leit, J. Rahil, A. J. Petschner, A. H. Lu, A. Nicolescu, S. Lefebvre, S. Montcalm, M. Fournel, T. P. Yan, Z. Li, J. M. Besterman, and R. Déziel, "Sulfamides as novel histone deacetylase inhibitors", Bioorg. Med. Chem. Lett., Vol. 19, pp. 336340, 2009.

[37] S. Manku, M. Allan, N. Nguyen, A. Ajamian, J. Rodrigue, E. Therrien, J. Wang, T. Guo, J. Rahil, A. J. Petschner, A. Nicolescu, S. Lefebvre, Z. Li, M. Fournel, J. M. Besterman, R. Déziel, and A. Wahhab, "Synthesis and evaluation of lysine derived sulfamides as histone deacetylase inhibitors", Bioorg. Med. Chem. Lett., Vol. 19, pp. 1866-1870, 2009.

[38] B. Chen, P. A. Petukhov, M. Jung, A. Velena, E. Eliseeva, A. Dritschilob, and A. P. Kozikowski, "Chemistry and biology of mercaptoacetamides as novel histone deacetylase inhibitors", Bioorg. Med. Chem. Lett., Vol. 15, pp. 1389-1392, 2005.

[39] O. Moradei, S. Leit, N. Zhou, S. Fre'chette, I. Paquin, S. Raeppel, F. Gaudette, G. Bouchain, S. H. Woo, A. Vaisburg, M. Fournel, A. Kalita, A. Lu, M. C. T. Bourget, P. T. Yan, J. Liu, Z. Li, J. Rahil, A. R. MacLeod, J. M. Bestermanb, and Daniel Delormea, "Substituted N-(2-aminophenyl)-benzamides, (E)-N-(2-aminophenyl)-acrylamides and their analogues: Novel classes of histone deacetylase inhibitors", Bioorg. Med. Chem. Lett., Vol. 16, pp. 4048-4052, 2006.

[40] I. Paquin, S. Raeppel, S. Leit, F. Gaudette, N. Zhou, O. Moradei, O. Saavedra, N. Bernstein, F. Raeppel, G. Bouchain, S. Fre'chette, S. H. Woo, A. Vaisburg, M. Fournel, A. Kalita, M. F. Robert, A. Lu, M. C. T. Bourget, P. T. Yan, J. Liu, J. Rahil, A. R. MacLeod, J. M. Besterman, Z. Lib, and Daniel Delorme, "Design and synthesis of 4-[(s-triazin-2ylamino)methyl]-N-(2-aminophenyl)-benzamides and their analogues as a novel class of histone deacetylase inhibitors", Bioorg. Med. Chem. Lett., Vol. 18, pp. 1067-1071, 2008. 